Published in final edited form as:

Curr Opin Infect Dis. 2016 June ; 29(3): 275-279. doi:10.1097/QCO.0000000000000258.

\title{
Varicella zoster virus and giant cell arteritis
}

\author{
Don Gilden ${ }^{\mathrm{a}, \mathrm{b}}$ and Maria A. Nagel ${ }^{\mathrm{a}}$ \\ aDepartment of Neurology, University of Colorado School of Medicine, Aurora, Colorado, USA \\ bDepartment of Immunology \& Microbiology, University of Colorado School of Medicine, Aurora, \\ Colorado, USA
}

\begin{abstract}
Purpose of review-Giant cell arteritis (GCA) is a serious disease and the most common cause of vasculitis in the elderly. Here, studies describing the recent discovery of varicella zoster virus (VZV) in the temporal arteries (TA) of patients with GCA are reviewed.
\end{abstract}

Recent findings-GCA is characterized by severe headache/head pain and scalp tenderness. Many patients also have a history of vision loss, jaw claudication, polymyalgia rheumatica, fever, night sweats, weight loss and fatigue. ESR and CRP are usually elevated. Diagnosis is confirmed by TA biopsy which reveals vessel wall damage and inflammation, with multinucleated giant cells and/or epithelioid macrophages. Skip lesions are common. Importantly, TA biopsies are pathologically negative in many clinically suspect cases. This review highlights recent virological findings in TAs from patients with pathologically-verified GCA and in TAs from patients who manifest clinical and laboratory features of GCA but whose TA biopsies are pathologically negative for GCA. Virological analysis revealed that VZV is present in most GCA-positive and GCA-negative TA biopsies, particularly in skip areas that correlate with adjacent GCA pathology.

Summary-The presence of VZV in GCA-positive and GCA-negative TAs reflects the possible role of VZV in triggering the immunopathology of GCA and indicates that both groups of patients should be treated with antivirals in addition to corticosteroids. Whether oral antiviral agents and steroids are as effective as intravenous acyclovir and steroids, as well as the dosage and duration of treatment, remain to be determined.

\section{Keywords}

varicella zoster virus; giant cell arteritis; temporal arteries

\section{INTRODUCTION}

VZV is an exclusively human neurotropic alphaherpesvirus. Primary VZV infection causes varicella (chickenpox), after which virus becomes latent in ganglionic neurons along the entire neuraxis. Decades later, as VZV-specific cell-mediated immunity wanes, virus

Correspondence: Don Gilden, MD, Department of Neurology, University of Colorado School of Medicine, 12700 E. $19^{\text {th }}$ Avenue, Box B182, Aurora, CO 80045, Telephone: 303-724-4326, don.gilden@ucdenver.edu.

Conflicts of interest

There are no conflicts of interest. 
reactivates, resulting in zoster (pain and rash restricted to 1-2 dermatomes). Unfortunately, the pain of zoster may persist for months or years (postherpetic neuralgia). Zoster may be further complicated by other serious neurological diseases such as meningoencephaliltis, cerebellitis, isolated or multiple cranial nerve palsies (polyneuritis cranialis), myelitis and vasculopathy, as well as multiple ocular disorders. These serious conditions cause paralysis, blindness and death. Importantly, all of the neurological and ocular disorders listed above can also develop in the absence of rash. The incidence and severity of zoster is best viewed as a continuum in immunodeficient individuals, ranging from a natural decline in VZVspecific immunity with advancing age to more serious host immune deficits encountered in organ transplant recipients and patients with cancer or AIDS.

VZV is the only human virus that has been shown to replicate in arteries and cause disease. Productive VZV infection in cerebral arteries leads to intracerebral VZV vasculopathy [1,2]. An exciting finding in the past few years is that productive VZV infection and vascular disease is not limited to the intracranial circulation; indeed, VZV infects extracranial TAs and is closely associated with GCA. The search for VZV in GCA was motivated by virtually identical pathological changes seen in patients with intracerebral VZV vasculopathy or with GCA. In both conditions, the pathology is characterized by granulomatous arteritis, in which inflammation, often transmural, is seen along with necrosis, usually in the arterial media; multinucleated giant cells, epithelioid macrophages or both are also present.

The association of granulomatous arteritis with the presence of VZV in intracerebral VZV vasculopathy prompted examination of TA biopsies for VZV from patients with pathologically-verified GCA and from patients with clinical features and laboratory abnormalities of GCA whose TA biopsies were pathologically-negative for VZV.

\section{CASE STUDIES}

The story began with virological analysis of a TA biopsy from an 80-year-old man who developed left ophthalmic-distribution zoster and ipsilateral ischemic optic neuropathy (ION) [3]. The TA revealed inflammation, but not the more extensive pathology characteristic of GCA. VZV antigen was abundant in the arterial adventitia and scattered throughout the media of the asymptomatic TA. The patient was initially treated with steroids but did not improve. After the presence of VZV was confirmed virologically, the patient was treated with intravenous acyclovir and his vision recovered. Overall, the patient was considered as having VZV-induced ION and subclinical TA infection. Importantly, the location of VZV in the TA suggested that virus entered arteries through nerve fibers within the adventitia and spread transmurally.

The second patient was a 75-year-old woman who developed periorbital pain and blurred vision OS. There was no history of zoster rash. Visual acuity was 20/40 OD, 20/400 OS, with a mild left relative afferent pupillary defect [4]. The left optic nerve was swollen and hyperemic with peripapillary flame hemorrhages. ESR was $124 \mathrm{~mm} / \mathrm{hr}$. She was treated with intravenous methylprednisolone, $250 \mathrm{mg}$ q6h. On day 3, headache and vision improved. ESR was $98 \mathrm{~mm} / \mathrm{hr}$ and CRP was $1.40 \mathrm{mg} \%$. Rheumatoid factor, ANA and ANCA titers were negative. On day 4, left TA biopsy was GCA-negative; steroids were changed to oral 
prednisone, $60 \mathrm{mg}$ daily. On day 7, brain MRI with gadolinium was negative. On day 9, pain and vision worsened. On day 11, orbital CT and head CT angiography were negative. On day 15 , visual acuity was 20/400 OS with relative left APD. On day 17, the OS became blind and non-reactive to light; fundus was obscured by vitreous hemorrhage. CSF contained $8 \mathrm{WBCs} / \mathrm{mm}^{3}$, protein $72 \mathrm{mg} \%$, glucose $54 \mathrm{mg} \%$. CSF cultures for bacteria, fungi, AFB and cytology were negative. Despite the absence of GCA pathology in the TA, VZV ischemic optic neuropathy (ION) was considered, and she was treated with intravenous acyclovir, 10 $\mathrm{mg} / \mathrm{kg} \mathrm{q} 8 \mathrm{~h}$ for 7 days. On day 31, CSF contained anti-VZV IgG but not anti-HSV IgG antibody, and serum-to-CSF ratio of anti-VZV IgG was reduced (14) compared to ratios for total IgG (121) and albumin (81). Immunohistochemistry and pathology revealed VZV antigen and neutrophils in the original left TA specimen. On day 31, she was treated with oral valacyclovir, $1 \mathrm{~g}$ TID for 6 weeks; prednisone was reduced to $20 \mathrm{mg}$ daily and tapered to $5 \mathrm{mg} /$ week. Six weeks later, pain resolved and visual acuity improved to finger-counting. The left optic nerve was pale, with clear margins and resolution of hemorrhage. Essentially, another case of VZV ION with sublinical TA infection was uncovered, this time with no history of zoster rash.

The next link of VZV vasculopathy with features of GCA was provided by a 54-year-old diabetic woman who developed ION followed by acute retinal necrosis and multiple areas of focal venous beading [5]. The vitreous fluid contained amplifiable VZV DNA but not HSV-1, CMV or toxoplasma DNA. The clinical presentation was remarkable for jaw claudication and intermittent scalp pain, prompting a TA biopsy that was pathologicallynegative for GCA but notable for the presence of VZV antigen. The case added to the clinical spectrum of multifocal VZV vasculopathy.

\section{DETECTION OF VZV IN GCA-POSITIVE AND -NEGATIVE TAS}

Further efforts to address the incidence of VZV infection in archived GCA biopsy-negative TAs from subjects with clinically suspected GCA revealed VZV, but not HSV-1 antigen, in 5/24 (21\%) TAs of these patients [6]. Thirteen normal TAs did not contain VZV or HSV-1 antigen. Interestingly, all 5 subjects whose TAs contained VZV antigen presented with clinical and laboratory features of GCA that included early visual disturbances. At that point, it was clear that multifocal VZV vasculopathy can present with the full spectrum of clinical features and laboratory abnormalities characteristic of GCA, and that in GCAnegative/VZV-positive TAs, viral antigen predominated in the arterial adventitia.

During the continuing search for VZV antigen in GCA-negative TAs, the TA of one subject revealed abundant VZV antigen and VZV DNA in multiple regions (skip areas) spanning $350 \mu \mathrm{m}$, as well as in skeletal muscle adjacent to the infected TA; pathological analysis of sections adjacent to those containing viral antigen revealed inflammation involving the arterial media and abundant multinucleated giant cells characteristic of GCA [7]. The detection of VZV followed by additional pathological studies led to a change in diagnosis for GCA-negative to classic GCA. In three other such cases, the detection of VZV in a GCA-negative TA led to more extensive pathological studies and also a change in diagnosis to GCA. 


\section{RETROSPECTIVE ANALYSIS OF ARCHIVED GCA-POSITIVE AND - NEGATIVE TAs FOR VZV}

To test the hypothesis that VZV infection triggers the inflammatory cascade characteristic of GCA, formalin-fixed, paraffin-embedded GCA-positive TA biopsies (50 sections/TA), including adjacent skeletal muscle, and normal TA biopsies from subjects $>50$ years of age were examined for the presence and distribution of VZV antigen by immunohistochemistry and ultrastructurally for virions; adjacent regions were examined by hematoxylin-eosin staining. Most VZV antigen-positive slides were analyzed by PCR for VZV DNA. VZV antigen was detected in 61/82 (74\%) GCA-positive TAs compared with 1/13 (8\%) normal TAs (p<0.0001). Most GCA-positive TAs contained viral antigen in skip areas [8 $\left.{ }^{\mathbf{m}}\right]$. VZV antigen, present mostly in the arterial adventitia followed by the media and intima, was detected in 12/32 (38\%) skeletal muscles adjacent to VZV antigen-positive TAs (Figure 1). Despite formalin fixation, VZV DNA was readily detected in: 18/45 (40\%) GCApositive/VZV antigen-positive TAs; 6/10 (60\%) VZV antigen-positive skeletal muscles; and $1 \mathrm{VZV}$ antigen-positive normal TA. VZ virions were also found in a GCA-positive TA. GCA pathology in sections adjacent to those containing VZV was seen in $89 \%$ of GCA-positive TAs, but in none of 18 adjacent sections from normal TA. Overall, most GCA-positive TAs contained VZV in skip areas that correlated with adjacent GCA pathology, strongly suggesting that VZV triggers GCA immunopathology.

The detection of well-delineated herpesvirus particles by transmission and scanning EM in the same area stained with anti-VZV antibody is further evidence of VZV in GCA-positive TAs. Moreover, despite fixation, VZV DNA was readily amplifiable in many slides that contained VZV antigen. Together, the findings of VZV antigen, VZV DNA and VZ virions in TAs indicate productive infection.

The search for VZV was extended to patients with clinically-suspect GCA with histopathologically-negative TA biopsies, as well as biopsy-positive and normal TAs removed postmortem from age-matched subjects [ $9^{\mathbf{m}}$ ]. VZV antigen was found in 45/70 (64\%) GCA-negative TAs compared with 11/49 (22\%) normal TAs ( $\mathrm{p}<.001$ ), and extension of our earlier study revealed VZV antigen in 68/93 (73\%) of GCA-positive TAs compared with 11/49 (22\%) normal TAs ( $\mathrm{p}<.001)$. Compared to the prevalence in normal TAs, VZV antigen was more likely to be present in both GCA-negative TAs ( $\mathrm{p}<.001)$ and GCA-positive TAs $(\mathrm{p}<.001)$. VZV was frequently found in perineurial cells expressing claudin-1 around nerve bundles. Of 44 GCA-negative subjects whose TAs contained VZV antigen, 16 (36\%) showed adventitial inflammation adjacent to viral antigen; no inflammation was seen in normal TAs. Together, our data suggest that the prevalence of VZV in the TAs of patients with clinically-suspected GCA is similar, independent of whether biopsy is negative or positive pathologically.

\section{NUMBER OF TA SECTIONS EXAMINED FOR VZV IS CRITICAL}

Success in detecting VZV in TAs was largely based on immunostaining of no less than 50 sections of every TA in all studies above. PCR in earlier studies to examine fixed GCApositive and -negative TAs for VZV did not reveal VZV DNA when 1-6 sections per TA 
were examined [10-13], and immunohistochemistry using VZV-specific antibodies did not detect VZV antigen when only one section from each TA was analyzed [10], whereas analysis of 4-6 sections detected VZV DNA in 9/35 (26\%) GCA-positive TAs [14], and examination of 10 sections detected VZV DNA in 18/57 (32\%) specimens from GCApositive TAs and in 18/56 (32\%) of GCA-negative TAs [15]. Furthermore, examination of 25 sections of each FFPE TA biopsy specimen revealed VZV antigen in 5/24 (21\%) GCAnegative TAs [6]. Overall, our detection of VZV antigen in most GCA-positive and GCAnegative TAs indicates the value of staining many sections, most likely increasing detection of virus in skip regions. Immunostaining of several hundred sections of each GCA-positive TA might reveal VZV in nearly all GCA-positive arteries.

\section{DISTRIBUTION OF VZV IN THE TA AND PRESENCE OF VZV IN SKIP AREAS INFORM PATHOGENESIS}

The consistently greater frequency of VZV in the adventitia than in the media and intima in GCA-positive and GCA-negative TAs most likely reflects transaxonal transport of virus along afferent nerve fibers that innervate the TA after reactivation from ganglia [16]. VZV in skip areas and almost always adjacent to pathology parallels the pathological features of GCA. The presence of VZV in skeletal muscle likely reflects the fact that the mammalian superficial TA is richly innervated and that nociception in connective tissue of the temporalis muscle is relayed by afferent fibers with cell bodies in the trigeminal ganglia from which VZV reactivates. Interestingly, about $40 \%$ of patients with GCA have a history of polymyalgia rheumatica. Since muscle biopsy is not usually performed in these patients, the frequency of VZV infection in peripheral skeletal muscle is unknown.

Another important finding in our studies is that the presence of VZV DNA and VZV antigen in $18 \%$ of control TAs without inflammation indicates that VZV reactivates subclinically in some people over age 50. VZV DNA is found in latently infected human ganglia and VZV expression is limited to expression of late VZV gene 63 RNA [17]. If VZV were latent in TAs, only VZV DNA would be found, not VZV late glycoproteins and VZ virions.

\section{FUTURE OF VZV ANTIGEN DETECTION IN GCA AND TREATMENT}

As with GCA pathology, VZV antigen and inflammation are usually patchy and detected in only some sections. Our research-focused evaluation is not practical for routine diagnostic work-up of TA biopsies and while immunohistochemical evaluation is worthwhile, a negative result should still consider that a negative biopsy does not rule out GCA or VZV reactivation.

As for treatment of GCA, no trials have yet been conducted to determine whether antivirals and steroids confer additional benefit to steroids alone. Although many GCA patients improve with steroids, reports are legion of GCA patients who not only fail steroids, but also develop more disseminated VZV vasculopathy and die $\left[1 \mathbf{m}^{\mathbf{m}}, 19 \mathbf{}\right]$. If VZV triggers the immunopathology of GCA, antiviral treatment is likely to confer additional benefit to corticosteroid-treated GCA patients, although the optimal antiviral regimen remains to be determined. We currently treat GCA with prednisone, $1 \mathrm{mg} / \mathrm{kg}$, along with valacyclovir, 1 
gm 3 times daily. If the patient improves after 4-6 weeks, we recommend tapering prednisone and thereafter administering antiviral reagents for another 4-6 weeks. Long-term antiviral drugs are far less risky than long-term steroids.

\section{CONCLUSION}

Virological analysis of TAs from patients with pathologically-verified GCA and TAs from GCA-negative patients manifesting clinical and laboratory features of GCA whose TA biopsies are pathologically-negative for GCA revealed the presence of VZV in most GCApositive and GCA-negative TA biopsies, particularly in skip areas that correlate with adjacent GCA pathology. The presence of VZV in GCA-positive and GCA-negative TAs reflects the possible role of VZV in triggering the immunopathology of GCA and indicates the need for treatment in both groups of patients with antiviral drugs in addition to corticosteroids. Whether oral antiviral agents and steroids are as effective as intravenous acyclovir and steroids, as well as the dosage and duration of treatment, remains to be determined.

\section{Acknowledgments}

We thank Marina Hoffman for editorial review and Cathy Allen for word processing and formatting.

Financial support and sponsorship

This work was supported in part by NIH grant AG032958 (D.G., M.A.N.), NS093716 (D.G.) and NS094758 (M.A.N.).

\section{REFERENCES AND RECOMMENDED READING}

Papers of particular interest, published within the annual period of review, are highlighted as:

of special interest

- of outstanding interest

1. Gilden DH, Kleinschmidt-DeMasters BK, Wellish BS, et al. Varicella zoster virus, a cause of waxing and waning vasculitis: The New England Journal of Medicine case 5-1995 revisited. Neurology. 1996; 47:1441-1446. [PubMed: 8960724]

2. Nagel MA, Cohrs RJ, Mahalingam R, et al. The varicella zoster virus vasculopathies: clinical, CSF, imaging, and virologic features. Neurology. 2008; 70:853-860. [PubMed: 18332343]

3. Salazar R, Russman AN, Nagel MA, et al. Varicella zoster virus ischemic optic neuropathy and subclinical temporal artery involvement. Arch Neurol. 2011; 68:517-520. [PubMed: 21482932]

4. Nagel MA, Russman AN, Feit H, et al. VZV ischemic optic neuropathy and subclinical temporal artery infection without rash. Neurology. 2013; 80:220-222. [PubMed: 23255829]

5. Mathias M, Nagel MA, Khmeleva N, et al. VZV multifocal vasculopathy with ischemic optic neuropathy, acute retinal necrosis and temporal artery infection in the absence of zoster rash. $\mathrm{J}$ Neurol Sci. 2013; 325:180-182. [PubMed: 23312850]

6. Nagel MA, Bennett JL, Khmeleva N, et al. Multifocal VZV vasculopathy with temporal artery infection mimics giant cell arteritis. Neurology. 2013; 80:2017-2021. [PubMed: 23635966]

7. Nagel MA, Khmeleva N, Boyer PJ, et al. Varicella zoster virus in the temporal artery of a patient with giant cell arteritis. J Neurol Sci. 2013; 335:228-230. [PubMed: 24125020] 
8ם. Gilden D, White T, Khmeleva N, et al. Prevalence and distribution of VZV in temporal arteries of patients with giant cell arteritis. Neurology. 2015; 84:1948-1955. The first large-scale search for VZV in archived GCA-positive TAs from 13 countries around the world. [PubMed: 25695965]

9ロ. Nagel MA, White T, Khmeleva N, et al. Analysis of varicella zoster virus in temporal arteries biopsy-positive and -negative for giant cell arteritis. JAMA Neurol. 2015; 72:1281-1287. The first large-scale search for VZV in both archived GCA-positive and -negative TAs for VZV. [PubMed: 26349037]

10. Nordborg C, Nordborg E, Petursdottir V, et al. Search for varicella zoster virus in giant cell arteritis. Ann Neurol. 1998; 44:413-414. [PubMed: 9749614]

11. Kennedy PG, Grinfeld E, Esiri MM. Absence of detection of varicella-zoster virus DNA in temporal artery biopsies obtained from patients with giant cell arteritis. J Neurol Sci. 2003; 215:27-29. [PubMed: 14568124]

12. Rodriguez-Pla A, Bosch-Gil JA, Echevarria-Mayo JE, et al. No detection of parvovirus B19 or herpesvirus DNA in giant cell arteritis. J Clin Virol. 2004; 31:11-15. [PubMed: 15288607]

13. Cooper RJ, D'Arcy S, Kirby M, et al. Infection and temporal arteritis: a PCR-based study to detect pathogens in temporal artery biopsy specimens. J Med Virol. 2008; 80:501-505. [PubMed: 18205226]

14. Mitchell BM, Font RL. Detection of varicella zoster virus DNA in some patients with giant cell arteritis. Invest Ophthalmol Vis Sci. 2001; 42:2572-2577. [PubMed: 11581201]

15. Alvarez-Lafuente R, Fernandez-Gutierrez B, Jover JA, et al. Human parvovirus B19, varicella zoster virus, and human herpes virus 6 in temporal artery biopsy specimens of patients with giant cell arteritis: analysis with quantitative real-time polymerase chain reaction. Ann Rheum Dis. 2005; 64:780-782. [PubMed: 15834059]

16. Gilden D, Nagel MA, White T, Grose C. WriteClick Editor's Choice: Is temporal arteritis due to VZV infection? Neurology. 2015; 85:1914-1915.

17. Ouwendijk WJD, Choe A, Nagel MA, et al. Restricted varicella zoster virus transcription in human trigeminal ganglia obtained soon after death. J Virol. 2012; 86:10203-10206. [PubMed: 22740396]

18. Nagel MA, Lenggenhager D, White T, et al. Disseminated VZV infection and asymptomatic VZV vasculopathy after steroid abuse. J Clin Virol. 2015; 66:72-75. This was an important exhaustive case study that revealed VZV in multiple organs and arteries in a patient who abused steroids. [PubMed: 25866342]

19 G. Gilden D, White T, Galetta SL, et al. Widespread arterial infection by varicella zoster virus explains refractory giant cell arteritis. Neurol Neuroimmunol Neuroinflamm. 2015; 2:e125. A retrospective analysis of a patient with GCA who failed treatment with steroids and died of disseminated VZV vasculopathy. doi: 10.1212/NXI.0000000000000125 [PubMed: 26090509] 


\section{KEY POINTS}

- $\quad$ VZV is the only human virus shown to replicate in arteries and cause disease.

- The pathology of intracerebral VZV vasculopathy and giant cell arteritis is characterized by granulomatous arteritis.

- $\quad$ Productive VZV infection (herpesvirus virions, VZV antigen and VZV DNA) is seen in intracerebral VZV vasculopathy and giant cell arteritis.

- The number of temporal artery sections examined for VZV is critical.

- $\quad$ The distribution of VZV in the temporal artery and the presence of VZV in skip areas inform pathogenesis.

- $\quad$ The detection of VZV in some normal temporal arteries indicates subclinical VZV reactivation, not another site of VZV latency.

- Because VZV likely triggers the immunopathology of giant cell arteritis, antiviral treatment may confer additional benefit to corticosteroid-treated patients, although the optimal antiviral regimen remains to be determined. 


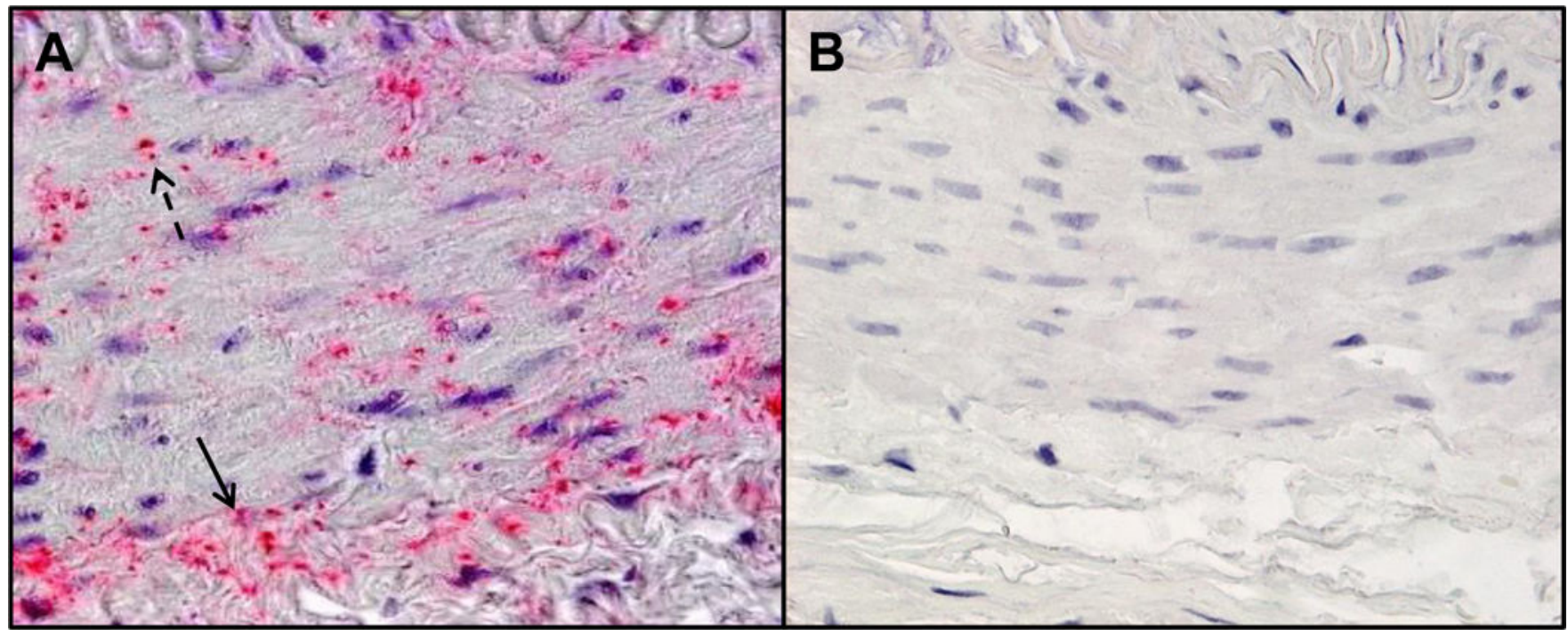

FIGURE 1.

Varicella zoster virus (VZV) antigen in the temporal artery (TA) from a patient with pathologically-verified giant cell arteritis (GCA). Immunohistochemical analysis of a GCApositive TA with mouse anti-VZV gE IgG1 antibody revealed VZV antigen in the adventitia (A, solid arrow) and media (A, dotted arrow) that was not seen when mouse isotype $\operatorname{IgG} 1$ control antibody (B) was substituted for mouse anti-VZV gE IgG1 antibody. 600x magnification. 